\title{
Desafíos de la inteligencia artificial en Odontología
}

\section{Carta al Editor}

\author{
Laura Galvis Zambrano 1,a, Carolina Pino Vélez 1,a \\ ${ }^{1}$ Universidad Santo Tomás de Aquino, Especialidad \\ Periodoncia, Bucaramanga, Colombia. \\ a Periodoncista.
}

\section{Correspondencia:}

Laura Galvis Zambrano: lamegaza@dentalnetla.net Universidad Santo Tomás sede Floridablanca. Carrera 27\#180-345, Floridablanca, Santander, Colombia ORCID: 0000-0001-5244-1228

\section{Coautor:}

\section{Challenges of artificial intelligence in dentistry}

Conflicto de intereses: ninguno.

Fuente de financiamiento: autofinanciado.

Recibido: $18 / 05 / 21$

Aceptado: 19/05/21

Publicado: 01/07/21

\section{Sr. Editor.}

Uno de los desafíos clave en la inteligencia artificial (IA) es cómo llevar el conocimiento informal a una computadora. El creciente desarrollo de la información, las bases de datos y las formas de conocimiento han hecho que se desarrollen tecnologías que ayuden a realizar tareas complejas en áreas de la Odontología. El papel de la IA dispone estas herramientas que aportan nuevas perspectivas para el diagnóstico, clasificación, pronóstico y la planificación del tratamiento odontológico. Por medio de la presente carta al editor se expone la importancia de la intervención de la IA en la práctica clínica odontológica.

De esta manera, el concepto de IA aplicado a la ciencia fue introducido por primera vez por el matemático John McCarthy en el año 1956, el cual posteriormente fue modificado por Richard Bellman quien consideró que la IA está asociada al pensamiento humano e incluye el aprendizaje y resolución de problemas. A su vez, la IA aplicada al campo de la Odontología ha permitido mejorar la precisión, lo que favorece mantener de forma digital los registros clínicos del paciente ${ }^{1}$. Así, en el año 2015, la IA llegó a entornos investigativos en el área odontológica, principalmente se empleó en las radiografías dentales y posteriormente en el campo clínico ${ }^{2,3}$. La IA se divide en dos tipos: machine learning y deep learning. El machine learning lo introdujo por primera vez $\mathrm{Ar}-$ thur Samuel en 1959, básicamente el machine learning permite que un computador tenga un aprendizaje automático lo que permite clasificar y predecir un resultado. Por otro lado, el deep learning permitió mejorar el machine learning y se aplica ampliamente en el campo de Odontología.

Un ejemplo muy común es con el uso de radiografías 2D digitales, en las cuales la reconstrucción de la imagen consta de miles de píxeles, estos píxeles pueden representar diferentes opacidades que corresponden a 
las densidades de las estructuras observadas, por lo que, mediante el deep learning, estas características (densidades representadas en píxeles) son aprendidas al analizar la imagen digital aplicando algoritmos que permiten su aprendizaje. Este proceso se realiza entre un ingeniero de sistemas experto y un odontólogo. Para enseñar al programa a través de deep learning se ingresan en el sistema numerosas radiografías $2 \mathrm{D}$ digitalizadas para realizar un entrenamiento al programa, posteriormente el odontólogo identifica y anota de forma manual los puntos de referencia anatómicos en la imagen, finalmente el ingeniero experto utiliza la información ya obtenida para iniciar el modelo de aprendizaje en el software y enseñar la habilidad de predecir la información. Ambos profesionales verifican la precisión en el aprendizaje del software y entre más información se introduzca, mejor aprendizaje tiene el software, lo que se evidencia en la exactitud de su predicción ${ }^{4}$ (Figura).

Con el uso regular de la IA a través de imágenes digitalizadas y registros médicos electrónicos, hasta ahora en el campo de visión computacional ${ }^{5}$, varios factores empiezan a ser relevantes:

- Es adecuada para superar la variabilidad en el examen de imágenes diagnósticas y aumentar la efectividad en el análisis mejorando el diagnóstico, la predicción y la toma de decisiones.

- Proporciona los datos de la salud digital de manera más limpia y estructurada.

- Permite la integración de datos de dominios diferentes y heterogéneos creando multiniveles que van a interactuar entre ellos.

- Facilita la investigación y descubrimiento complementando otros modelos existentes.

- Agiliza el trabajo rutinario de los profesionales permitiendo aumentar el tiempo cara a cara en la atención humanizadora con sus pacientes ${ }^{6}$.

- Promete hacer que la atención a pacientes sea más participativa.

- Recopilación de datos de manera continua ${ }^{7}$ que tendrá como ventaja el monitoreo no invasivo de la salud y su comportamiento, lo que permitirá una comprensión individual mucho más profunda de los factores y procesos de la enfermedad/patología.
- Los costos del diagnóstico y tratamiento pueden reducirse, ayudando a los sistemas de salud.

Bajo las apreciaciones anteriores se puede concluir que, la IA contemporánea sobresale en el uso del conocimiento formalizado y la extracción de un conjunto de información masiva, sin embargo, ésta no logra hacer asociaciones como un cerebro humano y solo puede realizar parcialmente la toma de decisiones complejas en un entorno clínico. La odontología únicamente digital no incorpora en la atención la prestación del servicio por parte de un profesional, en donde este debe aplicar su intuición clínica, la percepción y la empatía que son claves en la atención odontológica personalizada y profesional. Sin embargo, se sugiere que el deep learning pueda ser empleado como ayuda en el diagnóstico, pero es importante ser cauteloso a la hora de emplear dicha herramienta para este fin, puesto que hasta el momento la precisión en el diagnóstico oscila entre $82 \%-89 \%$ en los diferentes estudios publicados en los que se utiliza el deep learning con radiografías $2 \mathrm{D}$ digitalizadas, lo que indica que el juicio clínico siempre deberá anteponerse.

\section{Referencias bibliográficas}

1. Priyankar at all. Artificial intelligence in dentistry and it's future. GSCARR. 2021;07(01):082-086. DOI: 10.30574/gscarr.2021.7.1.0078

2. Topol E. Deep medicine: how artificial intelligence can make healthcare human again. New York: Basic Books. 2019. DOI: $10.1136 /$ bmj.m689

3. Lee JH, Kim D, Jeong SN, Choi SH. Diagnosis and prediction of periodontally compromised teeth using a deep learning-based convolutional neural network algorithm. J Periodontal Implant Sci. 2018;48(2):114. DOI: 10.5051/jpis.2018.48.2.114.

4. Yo-we $\mathrm{CH}$ te al. Artificial intelligence in dentistry: current applications and future perspective. Quintessence Int. 2020;51(3):248-257. DOI: 10.3290/j.qi.a43952.

5. Naylor CD. On the prospects for a (deep) learning health care system. JAMA. 2018;320(11):1099-100.

6. Israni ST, Verghese A. Humanizing artificial intelligence. JAMA. 2019;321(1):29-30.

7. Schwendicke F, Golla T, Dreher M, Krois J. Convolutional neural networks for dental image diagnostics: a scoping review. J Dent. 2019;91:103226. DOI: 10.1016/j. jdent.2019.103226.

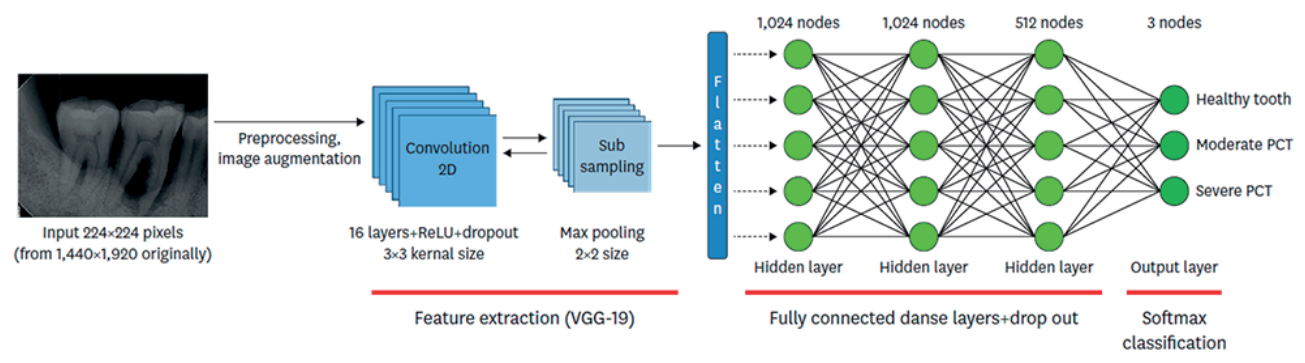

Figura. Ejemplo de modelo de CNN con deep learning, tomado estudio de Lee $^{3}$ 